\section{Chrischta Ganz}

\section{Warum dieser Kongress?}

Als einen der Gründe für den Kongress nannten die Organisatoren den «Ja»-Anteil von $67 \%$ in der eidgenössischen Volksabstimmung «Zukunft mit Komplementärmedizin» vom 17. Mai 2009.

Die Weltgesundheitsorganisation kennt folgende Definition:

«Die Begriffe Alternativmedizin/ Komplementärmedizin (CAM/Complementary and Alternative Medicine) umfassen ein breites Spektrum von Heilmethoden, die nicht Teil der Tradition des jeweiligen Landes sind und nicht in das dominante Gesundheitssystem integriert sind.»

Diese Erklärung zeigt, dass eine zukunftsorientierte Medizin die Einbindung der ganzheitlichen Betrachtungsweise der traditionellen Naturheilkunde und deren Systeme in das sogenannte «dominante Gesundheitssystem» verlangt.

Wo sind Verknüpfungen zwischen der jahrtausendealten, vielfältig entwickelten Naturheilkunde und der jungen, ungefähr 125 Jahre alten «wissenschaftlichen Schulmedizin» möglich? Wie kann die Naturheilkunde in die «Moderne» heraustreten bzw. die zukünftige Medizin mitgestalten? Wie kann der Dialog in der ganzheitlichen Medizin der Zukunft präsent und aktiv gestaltet werden? Um Antworten auf diese Fragen zu finden und um Berührungsängste zwischen den einzelnen Fachrichtungen abzubauen, hielten die beiden Kongresstage diverse Vorträge bereit.

Andréas Härry, der die beiden Kongresstage moderierte, begrüsste die Teilnehmenden am Samstagmorgen, stellte die Kongressorganisation (Thomas Bertschi und Heide-Dore

\title{
Nachbericht zum ersten Europäischen Kongress für Naturheilkunde, Alternativ- und Komplementärmedizin in Winterthur
}

Am 7. und 8. September 2013 fand in Winterthur der erste Europäische Kongress für Naturheilkunde, Alternativ- und Komplementärmedizin statt. Der vielversprechende Kongress war eine Plattform für eine zukunftsorientierte Medizin in Europa und begeisterte Fachpersonen aus den verschiedenen Richtungen der Komplementärtherapie ebenso wie Heilpraktiker, Naturärzte, Ärzte und Studierende der verschiedenen Ausbildungsinstitutionen. Das Thema des Kongresses lautete «Medizinalsysteme und Naturheilkunde» und umfasste sowohl Alternativ- und Komplementärmedizin als auch Schulmedizin und Naturwissenschaft. Den Kongressteilnehmenden wurde ein grosses Angebot an Fachreferaten zu Systemen und Methoden der Traditionellen Europäischen Naturheilkunde (TEN), zur Traditionellen Chinesischen Medizin (TCM), zur Homöopathie, zum Ayurveda und auch zur modernen Informationsmedizin von renommierten Referenten und Referentinnen aus der Schweiz, aus Deutschland und aus Österreich geboten.

Abb. 1. Die Kongressleitung Thomas Bertschi und Heide-Dore Bertschi-Stahl mit Heilpraktikerin Elvira Bierbach (von links nach rechts).

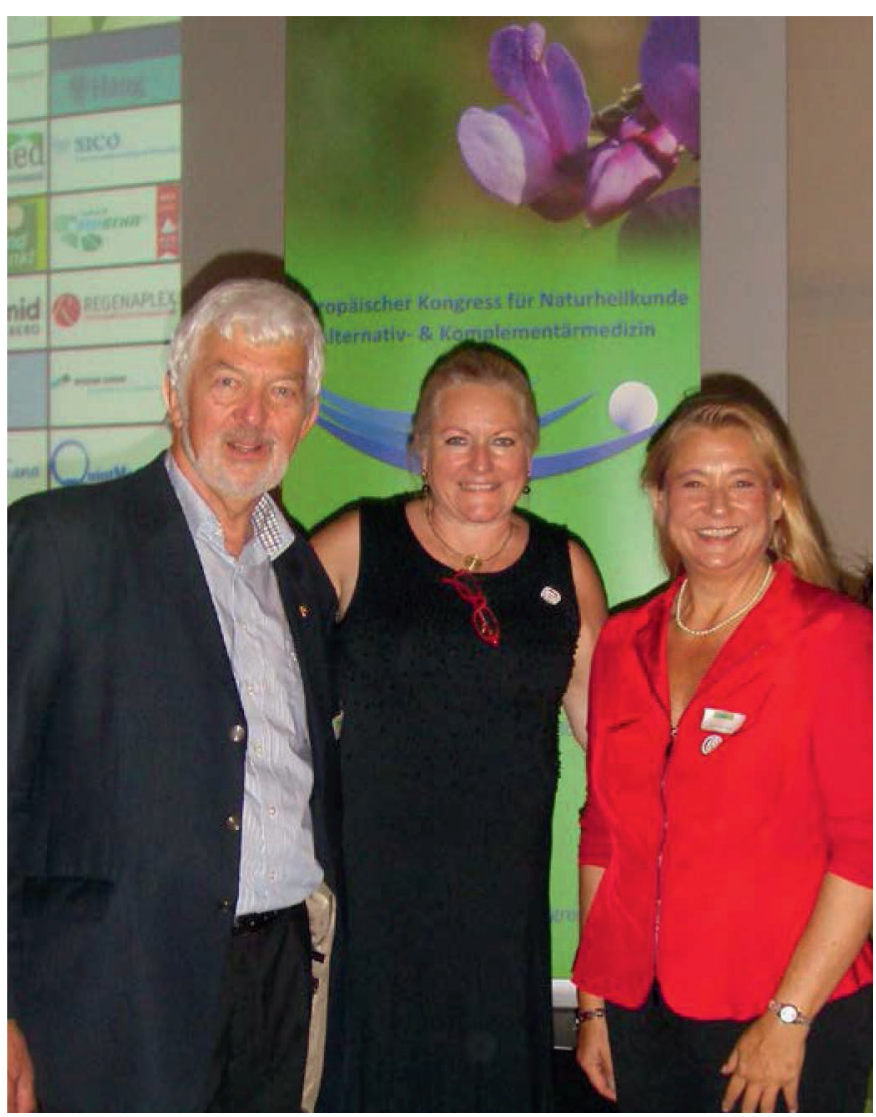

\section{KARGER}

Fax +497614520714 Information@Karger.com www.karger.com
(๔) 2013 S. Karger GmbH, Freiburg
Chrischta Ganz

Praxis für Naturheilkunde

Steinentischstrasse 1, 8002 Zürich, Schweiz

naturheilpraxis@chrischtaganz.ch 
Bertschi-Stahl, Abb. 1) sowie Michael Künzle, Stadtpräsident von Winterthur, und Hans Altherr, Ständerat des Kantons Appenzell Ausserrhoden, vor und leitete dann in den eigentlichen Kongress über.

\section{Eröffnungsreferat}

Elvira Bierbach (Abb. 1), Heilpraktikerin und Buchautorin aus Deutschland (unter anderem bekannt als Herausgeberin des Standardwerks «Naturheilpraxis heute»), beleuchtete in ihrem Referat die Frage, warum eine fundierte Ausbildung für Heilpraktiker und Heilpraktikerinnen notwendig ist - eine sehr aktuelle Frage, wenn man die Prozesse der Berufsbildung in der Schweiz verfolgt. Ebenso spannend bezüglich des Themas Berufsbildung in der Schweiz stellte Frau Bierbach zwei weitere Diskussionspunkte in den Raum:

i) Müssen bzw. sollen Experten einer Prüfungskommission selber Fachpersonen der Naturheilkunde sein?

ii) Inwieweit lässt sich die Akademisierung des Heilpraktikerberufs mit den ursprünglichen Wurzeln der Naturheilkunde vereinbaren, da ja wichtige Bereiche der Alternativmedizin immer schon aus volksheilkundlichem Wissen und aus der Erfahrungsmedizin gekommen sind?

Das Schlusswort von Frau Bierbach war ein schöner Auftakt in die Kongresstage: Naturheilpraktiker (oder genereller formuliert: Fachperson in der Ganzheitsmedizin) zu sein, sei der schönste Beruf der Welt, da der Mensch im täglichen Praxisalltag wirklich in der Mitte stehen kann, darf und soll.

\section{Die Vielfältigkeit der \\ Naturheilkunde}

Neben einer äusserst freundlichen und gleichzeitig höchst professionellen Atmosphäre zeigte der Kongress die Vielfältigkeit der Naturheilkunde in den verschiedensten Referaten auf. Nachfolgend eine Auswahl:

Dr. med. Florian Ploberger erzählte von seinen Erfahrungen mit westlichen Kräutern in der TCM. Als Fachmann sowohl für Traditionelle Tibetische Medizin (TTM) als auch für TCM verglich er verschiedene «blutbewegende» Pflanzen, wie sie in der europäischen Heiltradition ebenso bekannt sind.

Heilpraktiker Friedemann Garvelmann bot einen spannenden Einblick in die Kinderheilkunde mit Schwerpunkt Lymphatismus/Scrofulose aus der Sicht der TEN. Aufgrund seiner jahrzehntelangen Erfahrung als Heilpraktiker brachte er den Kongressteilnehmern die Thematik des fehlenden Wärmepotenzials und der sich daraus entwickelnden mangelhaften Effizienz der Abwehrtätigkeit näher. So erläuterte er die kompensatorischen Ersatzausscheidungen auf Schleimhaut (Katarrh) und Haut (Schleimhaut) sowie die Trias Lymphsystem, Schleimhaut und Darmflora. Auch betonte er die Bedeutung der Darmflora, allerdings differenzierte er in der konkreten Verabreichung von Darmsymbionten (kein Giesskannenprinzip bei Darmbakterien). So sind Erkrankungen wie Tonsillitis, Otitis media, Bronchitis usw. keine separat zu betrachtenden Erkrankungen, sondern lediglich verschiedene «Orte» und ein anderer «Ausdruck» derselben Problematik (Vikariation). Dies entspricht der Grundhaltung der Ganzheitsmedizin, dass nicht nur ein Symptom behandelt werden soll, sondern «alles mit allem zu tun hat» bzw. das System Mensch einer «systemischen» Behandlung bedarf.

Maja Dal Cero (dipl. nat. ETH) stellte ihr Fachgebiet «Heilpflanzentraditionen in der Schweiz» vor. Laut ihren Untersuchungen am Institut für Systemische Botanik der Universität Zürich werden von den geschätzten 2500 Pflanzen in der Schweiz heute noch etwa 200 als Heilpflanzen verwendet. In der Renaissance waren es noch mehr als 400 Medizinalpflanzen. Spannend war ihre Erkenntnis, dass 80-100 Heilpflanzen genannt werden können, die seit der Antike durch alle Epochen hindurch bis heute verwendet wurden/werden. Beispiele dafür sind unter anderem Taraxacum officinale (Löwenzahn), Foeniculum vulgare (Fenchel), Artemisia absinthium (Wermut), Urtica dioica (Brennnessel), Alchemilla millefolium (Schafgarbe) und Gentiana lutea (Gelber Enzian). Auch anhand der Pflanzenfamilien lassen sich die meistverwendeten Arzneipflanzen durch alle Epochen systematisieren: Apiaceae, Euphorbiaceae, Lamiaceae, Pinaceae, Rosaceae. Maja Dal Cero griff auch die Baumheilkunde auf - ein traditionell wichtiger Bestandteil der Heilkunde, der jedoch heute kaum mehr Bedeutung hat. Für die Zunahme und Abnahme der Anzahl der Heilpflanzen, die von der jeweiligen Bevölkerung verwendet wurde, stehen laut Maja Dal Cero nachfolgende Gründe im Vordergrund:

- Verfügbarkeit der Ressourcen (Bedarf/Nachfrage);

- Kostenbewusstsein/ Wirtschaftlichkeit;

- Qualitätsfragen;

- Entdeckung neuer Kontinente/Verstärkung von Handelsbeziehungen;

- Alternativen zu Heilpflanzen;

- Wahrnehmung/Definition von Arzneipflanzen («Ethnotaxa»). Maja Dal Cero schloss ihr Referat mit drei prägnanten Aussagen:

i) Ein ethnobotanischer Blickwinkel, der für eine Untersuchung der Arzneipflanzen in ihrem kulturellen Kontext sorgt, schafft eine Verbindung zwischen Naturwissenschaften und empirischem Wissen.

ii) Historische Bücher und schriftliche Quellen sind ein grosser Fundus an empirischem Arzneipflanzenwissen, das nach aktuellen wissenschaftlichen Standards geprüft werden kann.

iii) Der Begriff «Tradition» kann sich auf einen Zeitraum von mehr als 
2000 Jahren oder auf einige Jahrzehnte beziehen.

Globale Zusammenhänge stellte dann Martin Ott, Demeter-Landwirt der Schweiz, Fintan-Stiftung, Rheinau/Schweiz, und Präsident des Stiftungsrats des Forschungsinstituts für biologischen Landbau (FIBL), mit seiner Präsentation am Samstagabend vor.

Dass die Ernährung Teil der Gesundheit und die Verantwortung jedes Einzelnen sein muss, war nach dem Vortrag «Gender Food - Neuer Begriff für ein altes Phänomen» von Sybille Binder-Schai offensichtlich.

Dass das ökologische Gleichgewicht jedoch ebenso Thema sein soll, erklärte Ott den Kongressteilnehmern auf gut nachvollziehbare Weise. Er stellte seine These vor, dass der Mensch erst durch die Kuh sesshaft werden konnte. In seinem Buch «Kühe verstehen» kommentiert er den enormen Kraftfutterverbrauch (2,5 Tonnen pro Kuh und Jahr in Europa, in der Schweiz $700 \mathrm{~kg}$; Biomilchbauern verfüttern 300-400 kg Kraftfutter) folgendermassen: «Von diesen 2,5 Tonnen Kraftfutter könnten nötigenfalls fünf Menschen ein Jahr lang leben. Von dem, was die Kuh an menschlicher Nahrung aus den 2,5 Tonnen Getreide produziert (Milch, Fleisch, Käse usw.), kann aber nur ein Mensch leben. Mit anderen Worten: Füttert man die Kühe mit Menschennahrung aus dem Ackerbau, so vernichtet jede Kuh die Nahrung von vier Menschen.»

Gemäss Ott war die ursprüngliche Aufgabe einer Kuh, die Kleewiesen zu fressen, die man in der Fruchtfolge benötigte, um den Acker wieder in

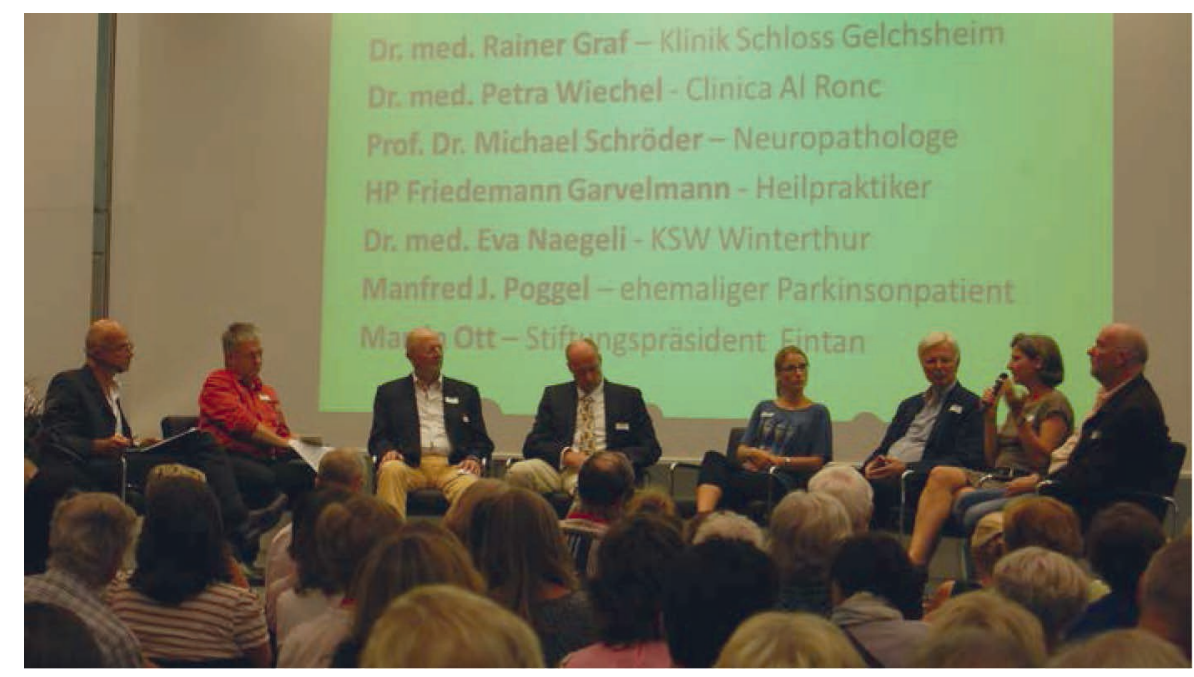

Abb. 2. Podiumsdiskussion am Ende des ersten Kongresstages.

Ordnung zu bringen und dabei die Erde mit den Ausscheidungen zu düngen. Landwirtschaft ist gemäss Ott nämlich als ein sich selbst erhaltendes System zu betrachten, sichtbar in der Kunst, den Boden in guter Qualität zu halten bzw. ihn zu verbessern - und ihn keineswegs auszubeuten, um möglichst viel zu produzieren. Die heutige Situation in der Agrarindustrie erzählt eine andere Geschichte.

An der Podiumsdiskussion (Abb. 2) am Samstagnachmittag stand die Frage «Wie sieht die Medizin von morgen aus?» im Mittelpunkt, wobei in die Diskussion auch aktuelle Themen wie das Epidemiengesetz (eidgenössische Volksabstimmung vom 22. September 2013) und die Impfproblematik miteinbezogen wurden. Zur Diskussion geladen waren Dr. med. Rainer Graf (Klinik Schloss Gelchsheim), Dr. med. Petra Wiechel (Clinica Al Ronc), Prof. Dr. Michael Schröder (Neuropathologe), Friedemann Garvelmann (Heilpraktiker), Dr. med.
Eva Naegeli (Kantonsspital Winterthur), Manfred J. Poggel (ehemaliger Parkinson-Patient) und Martin Ott (Stiftungspräsident Fintan, DemeterLandwirt). Durch das spannende und äusserst fesselnde Gespräch führte Andréas Härry. Martin Ott rückte dabei (wie auch in seinem eigenen Referat) die Gesundheit als eine Kraft der Kreativität und als ein Prinzip der Diversität in den Vordergrund, woraus sich zuallererst eines ergibt: Achtung vor jedem Individuum.

\section{Fazit und Ausblick}

Abschliessend bleibt zu sagen: Der erste Europäische Kongress für Naturheilkunde, Alternativ- und Komplementärmedizin war ein voller Erfolg. Der zweite Kongress - mit dem Thema «Jung und Alt» - ist bereits in Planung und wird in 2 Jahren, d.h. am 5. und 6. September 2015, stattfinden. 